\title{
DERMOLIPECTOMÍA ABDOMINAL FUNCIONAL EN PACIENTE DE ALTO RIESGO QUIRÚRGICO
}

\section{FUNCTIONAL ABDOMINAL DERMOLIPECTOMY IN PATIENT WITH HIGH SURGICAL RISK}

\author{
Alicia María Tamayo Carbón ${ }^{1}$, Williams Reynaldo Siñani Díaz ${ }^{2}$, Diego Darío García Torres ${ }^{3}$.
}

\section{RESUMEN}

La obesidad mórbida aparece como estadío final de la obesidad. Es una enfermedad crónica de origen multifactorial caracterizada por acumulación excesiva de grasa o hipertrofia del tejido adiposo, ocasionando problemas higiénicos, discapacidad funcional, afectando la calidad de vida. Se presenta paciente masculino de 66 años de edad, con panículo adiposo colgante severo, llegando a ambas rodillas con afectación cutánea, limitación funcional, movilización dependiente y discapacidad para realizar actividades diarias, siendo un paciente con alto riesgo y lipodistrofia abdominal severa. Se realizó Dermolipectomía Abdominal Funcional con resección de 20 kg de tejido dermograso. No presentó complicaciones y se logró su reincorporación social.

La Dermolipectomía Abdominal Funcional constituye la única opción para el tratamiento de pacientes con vientre péndulo. Aplicando cuidados perioperatorios adecuados se logra disminuir el número de complicaciones y se reintegra socialmente a un paciente minusválido que, a causa del enorme faldón abdominal estuvo limitado por muchos años.

\section{ABSTRACT}

Morbid obesity appears as final stage of obesity. It is a chronic disease of multifactorial origin characterized by excessive accumulation of fat or hypertrophy of adipose tissue, causing hygienic problems, functional disability, affecting the quality of life. It is presented a male patient of 66 years old, with severe hanging panniculus adipose, reaching both knees with skin involvement, functional limitation, dependent mobilization and disability to perform daily activities, is a patient with high risk and severe abdominal lipodystrophy. Functional Abdominal Dermolipectomy was performed with resection of $20 \mathrm{~kg}$ of dermo-fat tissue. There were no complications and his social reincorporation was achieved.

Functional Abdominal Dermolipectomy is the only option for treatment of patients with a pendulum belly. By applying adequate perioperative care, the number of complications is reduced and socially reintegrated into a handicapped patient who, due to the enormous abdominal skirt, was limited for many years.

\section{INTRODUCCIÓN}

T a obesidad es la enfermedad crónica de origen Lmultifactorial que afecta a la sociedad moderna, es el quinto factor principal de riesgo de defunción en el mundo. La obesidad mórbida es la forma más temible del sobrepeso pues además de disminuir la expectativa de vida causa discapacidad, minusvalía y problemas de exclusión social. Por sus efectos a nivel colectivo es un problema de salud pública en muchos países desarrollados como en vías de desarrollo como efecto de los cambios en las costumbres sociales y alimentarias $^{1,2}$.

$\mathrm{Ni}$ la dieta ni la cirugía bariátrica aislada son capaces de reducir la lipodistrofia abdominal una vez establecida. Teniendo en cuenta la clasificación de
Igwe (tabla 1)nos ayuda a cuantificar la extensión del mismo, evaluar y dar recomendaciones quirúrgicas (agradecimiento a la revista Obesity Surgery).

\section{PRESENTACIÓN DEL CASO}

Paciente masculino de procedencia rural, de 66 años de edad, con antecedentes de Hipertensión Arterial y Diabetes Mellitus tipo II controladas con tratamiento farmacológico; Enalapril $20 \mathrm{mg} /$ día y Metformina $850 \mathrm{mg} /$ día respectivamente con 123 $\mathrm{kg}$ de peso e Indice de Masa Corporal (IMC): 50 $\mathrm{kg} / \mathrm{m} 2$, quien acudió a consulta externa remitido del servicio de Cirugía general del Hospital Clínico Quirúrgico Hermanos Ameijeiras de La Habana, Cuba, para valoración reconstructiva por presentar faldón abdominal gigante incrementado tras pérdida
Especialista de II grado en Cirugía Plástica Reconstructiva y Quemados.

${ }^{2}$ Residente de 3er. Año en Cirugía Plástica Reconstructiva y Quemados.

${ }^{3}$ Residente de 2 do. Año en Cirugía Plástica Reconstructiva y Quemados.

Servicio de Cirugía Plástica Reconstructiva y Quemados. Hospital Clínico Quirúrgico Hermanos Ameijeiras. La Habana, Cuba.

Correspondencia a:

Williams Reynaldo Siñani Díaz Correo electrónico: williamssdg14@hotmail.com Celular: +53 54740888

Palabras clave: Dermolipecto mía funcional, obesidad, lipodistrofia abdominal

Keywords: Functional dermolipectomy, obesity, abdominal lipodystrophy

Procedencia y arbitraje: no comisionado, sometido a arbitraje externo.

Recibido para publicación: 9 de mayo de 2018 Aceptado para publicación 04 de junio de 2018

Citar como:

Rev Cient Cienc Med 2019; 22(1): 43-47 
Tabla 1. Clasificación de Igwe para colgajo abdominal redundante.

\begin{tabular}{|ll|}
\hline & CLASIFICACIÓN DE IGWE \\
Grado I & Llega al vello púbico. \\
Grado II & Cubre el pubis. \\
Grado III & Llega al tercio superior del muslo. \\
Grado IV & Cubre la mitad del muslo. \\
Grado V & Se extiende a la rodilla o por debajo de ella. \\
Fuente: Igwe D, Stanczyk M, LeeH. Panniculectomy adjuvant to obesity surgery. Obes Surg. 2000;10:530
\end{tabular}

masiva de peso de forma continua y progresiva, durante 5 años de evolución, luego de establecer un régimen dietético saludable. El paciente refería problemas con su higiene personal y se movilizaba en silla de ruedas con limitación funcional para sus actividades diarias.

El examen físico abdominal mostró a la inspección: abundante panículo adiposo, piel de naranja, edema y vergeturas. Auscultación y percusión sin alteraciones. Palpación: blando, depresible, sin tumoraciones, ni dolor. Mensuraciones: Test Rolling: positivo. Test Illouz: Negativo. Test Pinch: $>9 \mathrm{~cm}$. Diámetro abdominal: $140 \mathrm{~cm}$. Altura ombligo-pubis: $15 \mathrm{~cm}$. (Fig. 1) Se realizó ultrasonido abdominopélvico para descartar hernia, que impresionó la atrofia muscular abdominal sin eventración. Hemoquímica sanguínea: dentro de límites normales.

Se realizó una Dermolipectomía Abdominal Funcional. Indicaciones pre quirúrgicas: profilaxis antibiótica y antitrombótica, sedación y control dietético. Valoración por cardiología, cirugía general y anestesiología. Planificación preoperatoria, Acto quirúrgico: previa sepsia y antisepsia, bajo anestesia general, se realiza exéresis en bloque del faldón abdominal excedente, sin plicatura biaxial de rectos abdominales ni transposición umbilical, hemostasia rigurosa, cierre por planos previa colocación de dos drenajes de succión negativa, colocación de faja abdominal(Fig. 2). Tiempo quirúrgico dos horas. Indicaciones postoperatorias: control de signos vitales, hidratación, analgesia, control dietético, antibioticoterapia, deambulación precoz, medidas antitrombóticas, fisioterapia respiratoria y cuidados generales de enfermería.

Evolución: satisfactoria, sin complicaciones. Alta hospitalaria al quinto día postoperatorio con menos de $25 \mathrm{ml}$ de contenido serohemático en los drenajes. Seguimiento y control por consulta externa semanal. Retiro de puntos a los 21 días. Alta a los 30 días. (Fig. 3)

\section{DISCUSIÓN}

Los pacientes con obesidad mórbida que experimentan pérdida masiva de peso pueden llegar a presentar una masa abdominal que en los casos más graves ocasiona discapacidad, al punto de no poder deambular, como ocurre en el presente caso que se movilizaba en sillón de ruedas. Esta deformidad abdominal también es denominada Panniculusmorbidus, lipodistrofia abdominal gigante o elefantiasis abdominal ${ }^{2,4}$. La Dermolipectomía abdominal funcional es la técnica indicada en estos pacientes para eliminar el tejido excedente. El término Dermolipectomía habitualmente se asocia a la cirugía estética, pero el hecho que sea funcional la limita a la paniculectomía solamente sin tratar la pared muscular

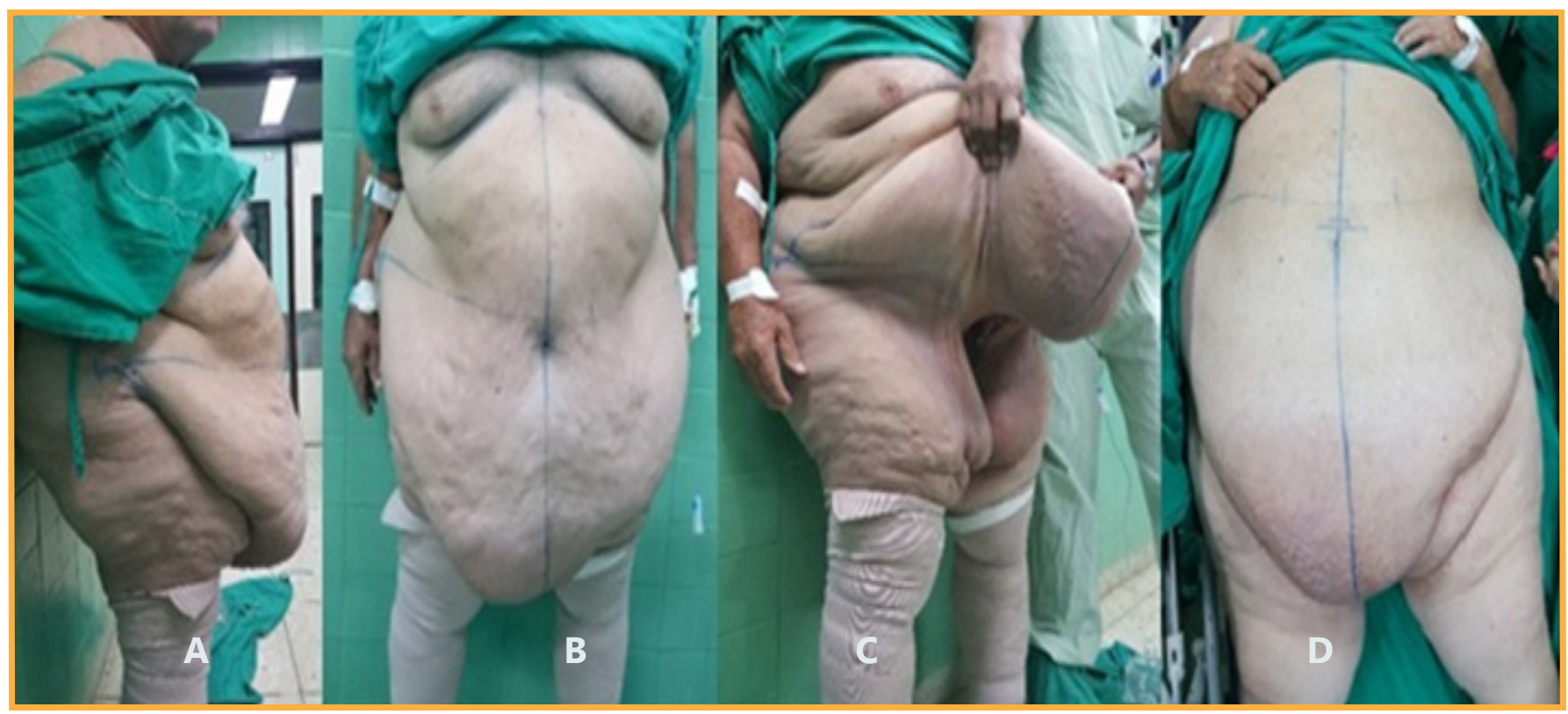

Figura 1: Vistas lateral (A), frontal (B), oblicua $(C)$ y en decúbito supino (D) que muestran extensión del faldón abdominal 
y sin aumentar la presión intraabdominal ${ }^{2,5,6,7}$.

La Dermolipectomía funcional al igual que la paniculectomía se presentan como las únicas opciones terapéuticas capaces de mitigar esa evolución desfavorable y permiten al paciente iniciar un programa de pérdida de peso $^{1,8,9}$ Ni la dieta ni la cirugía bariátrica aislada son capaces de reducir la lipodistrofia abdominal una vez establecida. Se trata de un procedimiento de alto riesgo por presentar numerosas comorbilidades, restricción en el flujo sanguíneo, dificultad respiratoria y heridas muy grandes, elementos que hacen muy difícil su manejo. Según a la bibliografía consultada, el índice de complicaciones está por encima del 50
$\%^{2,7}$, entre las más frecuentes están relacionadas con la herida quirúrgica como ser: seroma, hematoma, celulitis, infección y necrosis. A su vez son también frecuentes e importantes las pérdidas sanguíneas y las alteraciones hidroelectrolíticas y en menor frecuencia pero con alta tasa de mortalidad el tromboembolismo pulmonar, infarto agudo de miocardio y sepsis ${ }^{2,4}$.

De acuerdo con la clasificación de $\operatorname{Igwe}^{2,3}$, este paciente es Grado $\mathrm{V}$ donde el péndulo abdominal se extiende hasta la rodilla (Fig.1) y tiene un IMC de $50 \mathrm{~kg} / \mathrm{m}^{2}$. Mediante los Test Rolling o de rodamiento abdominal, Illouz o de contractura abdominal negativos en este caso por presentar gran diástasis abdominal y Pinch test o de pinzamiento abdominal

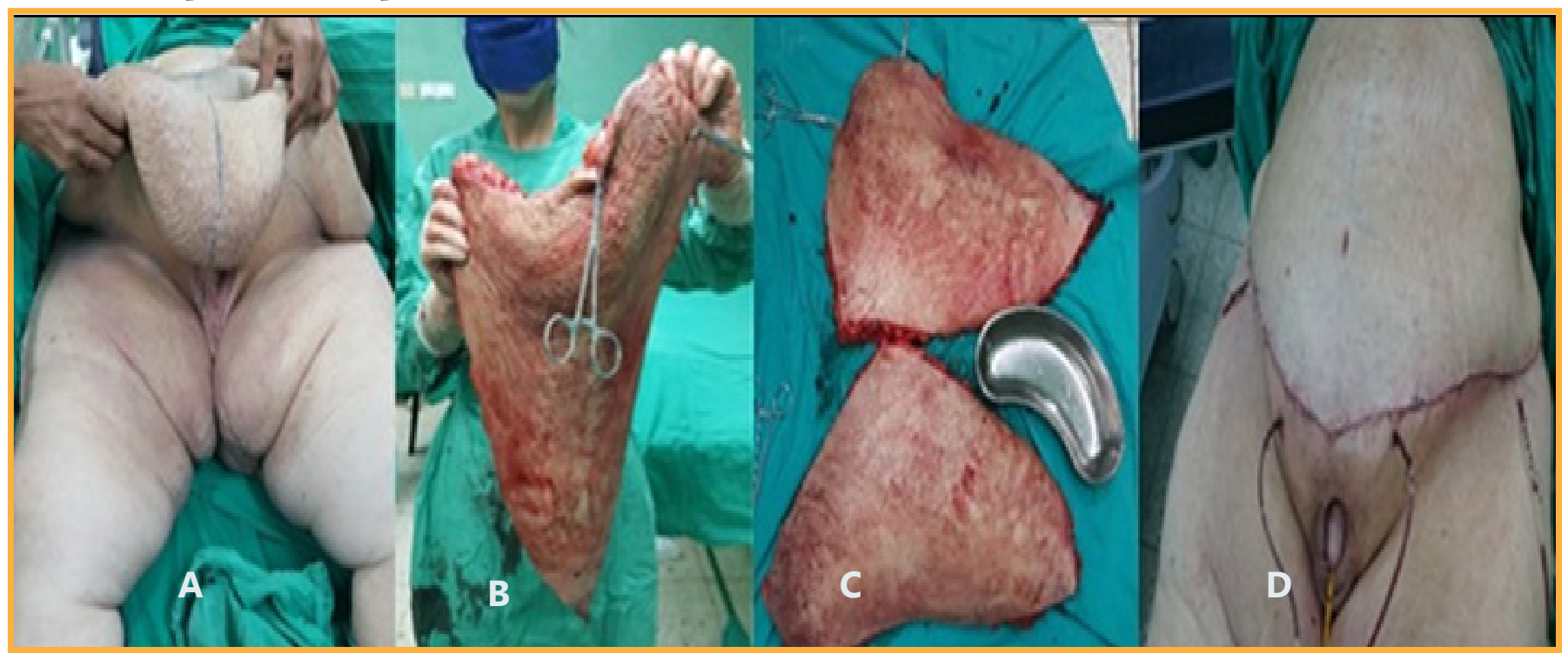

Figura 2: Pieza quirúrgica resecada $(A)$, dimensiones $(B$ y $C)$ y resultado postoperatorio inmediato $(D)$.

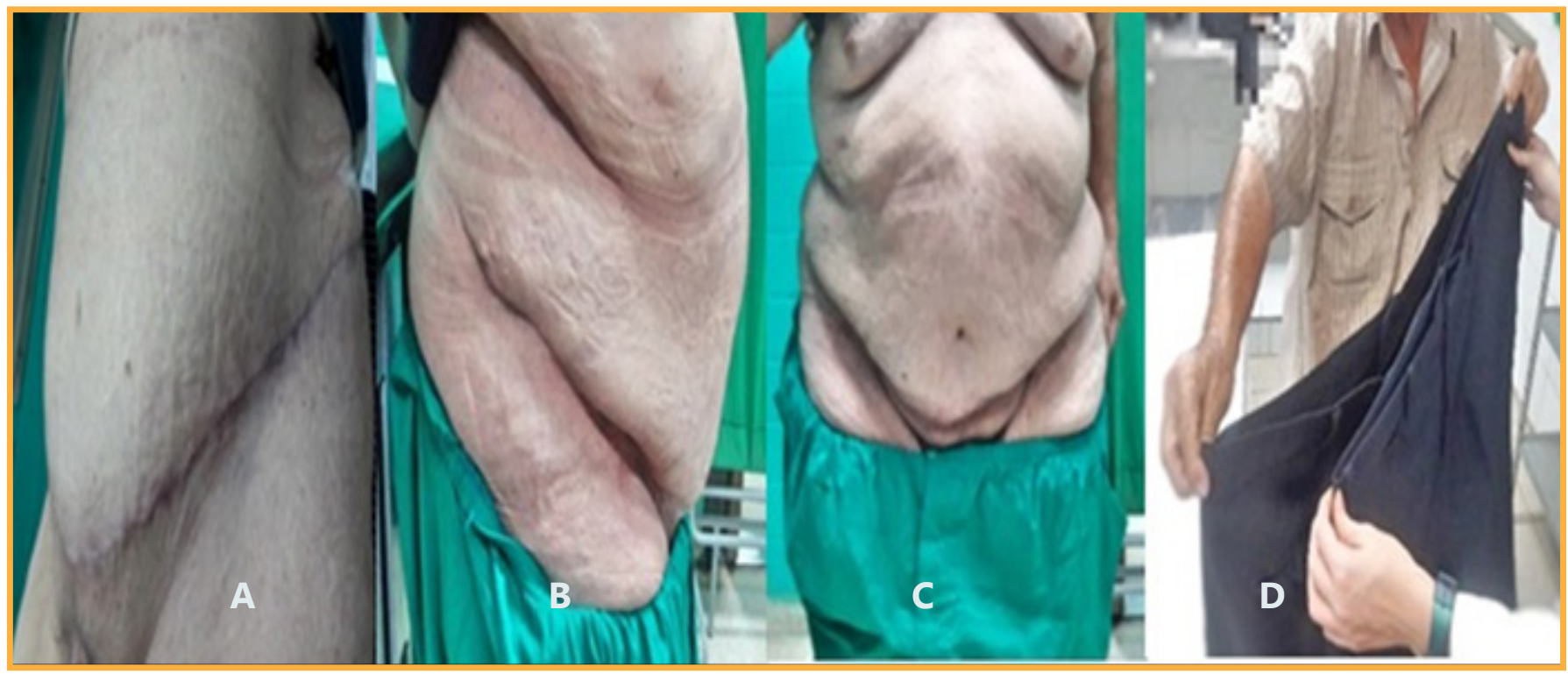

Figura 3: Postoperatorio a los 21 días aún sin retirar suturas (A). Vease la ausencia de zonas cruentas o granulomas. Vistas lateral (B) y frontal (C) a los 30 dias postoperatorios. Paciente mostrando reducción del perímetro abdominal a los tres meses (D).

Dermolipectomía abdominal funcional en paciente de alto riesgo quirúrgico. 
el cual $>2 \mathrm{~cm}$ es positivo, útiles en el examen físico para el diagnóstico y a la vez para la planificación quirúrgica. En aquellos casos más severos (Grado IV y V) la Dermolipectomía se realiza más con un interés funcional que estético (Fig.2).

La Dermolipectomía multifuncional en ancla ha demostrado ser una técnica altamente efectiva en el tratamiento de pacientes con grandes pérdidas ponderales, tanto en los que han sido tratados con técnicas de cirugía bariátrica, como en aquellos que han logrado adelgazar mediante tratamiento médico y medidas higiénico-dietéticas.

En pacientes con adelgazamiento moderado o grande, la dermolipectomía multifuncional consigue en un único tiempo quirúrgico una mejora multivectorial del contorno corporal; no sólo trata el faldón abdominal anterior, sino que permite una mayor redefinición de los flancos, del pubis y del tercio superior de muslos. Este tipo de técnica, aborda además del tratamiento del excedente cutáneo-graso resultante de la pérdida masiva de peso, también el plano músculo-aponeurótico ${ }^{10}$.

Sin embargo, como nuestro paciente presentaba alto riesgo quirúrgico se hizo énfasis sólo en lo funcional para asegurar una mejor evolución sin desarrollar complicaciones locales ni sistémicas, por tales motivos solo se reseco el tejido dermograso excedente. Cuanto más funcional es la cirugía, mayor es el grado de satisfacción del paciente. Los pacientes a los que hemos realizado esta técnica, buscan una mejora funcional y no sólo estética ${ }^{11}$.

Existen estudios que demuestran mayor porcentaje de complicaciones con un IMC $>39 \mathrm{~kg} / \mathrm{m}^{2} 2,8,12$. Consideramos que el éxito en este paciente con IMC: $50 \mathrm{~kg} / \mathrm{m}^{2}$ está en relación con los estrictos cuidados perioperatorios quirúrgicos y anestésicos.

La Dermolipectomía abdominal funcional debe realizarse en pacientes que mantienen una estabilidad ponderal de al menos un año y que además de un examen físico minucioso en busca de hernias ocultas (descritas en un $20 \%$ de los casos) se debe complementar con una Tomografía Axial Computarizada (TAC)si esta prueba de imagen no fuera posible se sugiere programar la intervención quirúrgica con el servicio de cirugía general ${ }^{1,2}$.

En el presente caso el paciente llega remitido de cirugía general con estudio completo que descartaba la presencia de hernias o evisceración. En la (Fig. 3) se observa el postoperatorio a los 21 días. Véase la ausencia de zonas cruentas o granulomas, con un cambio radical en la calidad de vida en cuanto a lo funcional, psicológico y social del paciente después de haberle realizado resección de $20 \mathrm{~kg}$ de tejido dermograso, constituyendo la mayor pieza quirúrgica resecada por el servicio implicado.

\section{CONCLUSIONES:}

La Dermolipectomía Abdominal Funcional a pesar de ser una cirugía de muy alto riesgo, constituye una buena opción para el tratamiento de pacientes con vientre péndulo. Debe ser considerado en esencia como un procedimiento reconstructivo $y$ de rehabilitación, que busca objetivos funcionales, psicológicos y mejoría en el aspecto físico. Se demuestra con este caso que aplicando todos los cuidados perioperatorios se logra que disminuya el número de complicaciones y se reintegra socialmente a un paciente minusválido que, estuvo limitado por muchos años a causa del enorme faldón abdominal. 


\section{REFERENCIAS}

1.Richter DK, Lampe H, Wolters M. Panniculectomy in patients with super obesity. En: Rubin P, Richter D, Uebel CO, JewellML, editores. Body contouring and liposuction. United States: Elsevier Saunders; 2013.. 265-77. DOI. 10.1097/01.prs.0000436818.34332.34

2. Esteban-Vico JR, Simón-Sanzb E, Delgado-Ruiza T, García Sáncheza JM, Llinás Portea A. Paniculectomía masiva en lipodistrofia abdominal gigante: cuando el beneficio supera los riesgos, rev hispanoam hernia. 2016; 4(4):173-178 S.L.U URL. https://www.sciencedirect.com/ science/article/pii/S2255267716000037

3. Igwe D, Stanczyk M, Lee H. Panniculectomy adjuvant toobesity surgery. Obes Surg. 2000; 10(6):530-9. URL https://www.ncbi.nlm.nih.gov/pubmed/11175961

4. Evans C, DeBord J, Howe H, Marshall JS, Rossi T, Owolabi M.Massive panniculectomy results in improved functionaloutcome. Am J Surg. 2014; 207:441-4. URL https://www.ncbi.nlm.nih.gov/pubmed/24439157

5. Friedrich JB, Petrov RV, Wiechmman SA, Clark MP, Foy HM,Isik FF, et al. Resection of Panniculus morbidus: A salvageprocedure with a steep learning curve. Plast Reconstr Surg.2008; 121:108-14. DOI:10.1097/01. prs.0000293760.41152.29

6. Semer NB, Ho WC, Mills S. Abdominal lipectomy: Aprospective outcomes study. Perm J. 2008; 12:23-6. URL https://www.ncbi.nlm.nih.gov/pubmed/18176212

7. Manahan MA, Shermak MA. Massive Panniculectomy aftermassive weight loss. Plast Reconstr Surg. 2006;
117: 2191-9. Disponible en https://www.ncbi.nlm.nih.gov/ pubmed/16772916

8. Zannis J, Wood BC, Griffin LP. Outcome study of the surgicalmanagement of panniculitis. Ann Plast Surg. 2012; 68:194-7. Disponible en https://www.ncbi.nlm.nih. gov/pubmed/21629099

9. Bonnet A, Mulliez E, Andrieux S, Duquennoy-Martinot V,Guerreschi P. Suspension of abdominal apron in massivepanniculectomy: A novel technique. J Plast Reconstr AesthetSurg. 2015; 68:272-73. Doi10.1016/j. bjps.2014.10.005

10. Muñoz V, Ayestaran S, Carames E. "Abdominoplastia funcional en ancla: nuestra experiencia $y$ nueva sistematica de abordaje en el paciente sin adelgazamiento" Cir. plást. iberolatinoam. 2010, 36 URL:http://scielo.isciii.es/scielo.php?script $=$ sci_ arttexte pid=S037678922010000400001

11. Sarwer, D.B.: "Psychiatric considerations of the Massive Weight loss Patient". Clin. Plastic. Surg. 35 (2008) 1. URL: https://www.plasticsurgery.theclinics.com/ article/S0094-1298(07)00136-8/pdf

12. Neaman KC, Hansen JE. Analysis of complications from abdominoplasty. Ann Plast Surg. 2007; 58:292-8.URL:https://doi.org/10.1097/01. sap.0000239806.43438.54 